International Journal of Advanced Trends in Computer Science and Engineering

Available Online at http://www.warse.org/IJATCSE/static/pdf/file/ijatcse951032021.pdf

https://doi.org/10.30534/ijatcse/2021/961032021

\title{
Smart Electronic Bin Using IoT
}

\author{
Chris Antony Correya ${ }^{1}$, Dixon Sebastian ${ }^{2}$, Stevin Felix ${ }^{3}$, Vishnu Dhanapal ${ }^{4}$, Renjini $\mathbf{H}^{5}$ \\ ${ }^{1}$ Department of Computer Science and Engineering, AISAT, Kalamassery, chrisantonycorreya100@ gmail.com \\ ${ }^{2}$ Department of Computer Science and Engineering, AISAT, Kalamassery, dixonsebastian369@ gmail.com \\ ${ }^{3}$ Department of Computer Science and Engineering, AISAT, Kalamassery, stevin.felix9@ gmail.com \\ ${ }^{4}$ Department of Computer Science and Engineering, AISAT, Kalamassery, vdhanapa1007@gmail.com \\ ${ }^{5}$ Department of Computer Science and Engineering, AISAT, Kalamassery, renjini.h@aisat.ac.in
}

\begin{abstract}
The amount of garbage that humans generate is rapidly increasing, and it will be impossible to control without radical adjustments. Such increases will have a significant impact on waste management firms, since they would be required to supply resources for garbage collection with little or no money. The goal of this project is to create a smart real-time waste controller system using the conceptual model of a smart electronic bin, which can be built by maximizing resource efficiency and optimizing the resources. This smart garbage bin prototype can automatically open the lid when it recognizes persons who wish to dispose of their trash. Even if people wish to dispose of their trash, if the waste bin is full, the lid will not open. It can also segregate plastic and non-plastic wastes. This smart bin is equipped with additional components such as GPS and GSM, for showing the location and to send alert messages. It can also detect the amount of trash in the garbage bin. The percentage-level of waste inside the bin is determined using an ultrasonic sensor. This data is delivered to a cloud-based monitoring and analytics IoT platform.
\end{abstract}

Key words: Internet of Things(IoT), Municipal Solid Waste(MSW), Global System for Mobile Communications(GSM), Smart Garbage System(SGS), Smart Garbage Bins(SGB), Global Positioning System(GPS), Radio Frequency Identification(RFID), Infrared(IR), Passive Infrared(PIR), Wireless Fidelity(Wi-Fi), Electrically Erasable Programmable Read-only Memory(EEPROM), Structured Query Language(SQL), General Packet Radio Service(GPRS).

\section{INTRODUCTION}

Large-scale waste generation and disposal has been a growing source of concern for the world, negatively impacting human lives and environmental situations. Wastes are those that grow in tandem with the country's development. Waste segregation is critical for environmentally responsible disposal of the large amounts of garbage that modern society generates. People have become accustomed to discarding items without considering the effects of their actions. Uncontrolled, unplanned, and unprotected dumping of industrial wastes at river sites and open areas is a typical form of disposal. This method will prove harmful to plants, human health and animal life.

In most nations, curbside collection, in which waste is collected at regular intervals by specialized trucks, is the most frequent method of disposal. The waste is subsequently transported to a proper disposal location. Waste collection services are currently overburdened in countries with developing economies. In such countries, waste collection methods are a constant issue, and many people struggle as a result of poor institutions and increased urbanization.

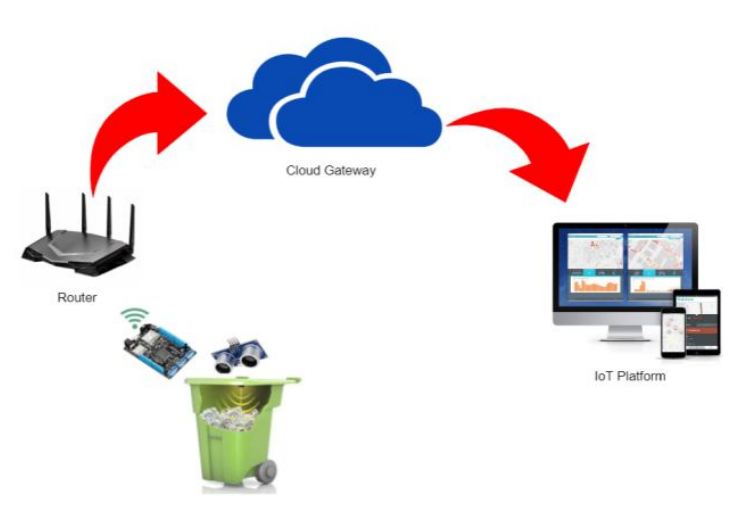

Figure.1: IoT-based Communication System

With the rise in population, economic upheaval, industrial expansion and changes in urban population, consumption patterns and lifestyles, the volume of municipal solid waste (MSW) generated is fast increasing. It became a huge challenge for the waste management authority to manage MSW. A significant portion of the total MSW management budget is spent on waste collection and transportation due to a lack of proper management options. As a result, in recent years, there has been a pressing need to improve the solid waste management system, which necessitates a wellorganized and proper method of monitoring the state of solid waste bins in real time while ensuring a green environment and society's viability. 
Smart cities have been highlighted as a promising prospective Internet of Things (IoT) application domain, with a wide range of services that can benefit both city management and inhabitants. Smart waste management is one service that could be offered in a smart city. Smart waste containers are required in smart cities. When public trash bins are overflowing for an extended period of time, they detract from the surrounding environment. On the other hand, sending a garbage truck to every trash bin in the city can be an expensive process; if the bins are empty, the trip is pointless. Cities create rudimentary algorithms to reduce the cost of various municipal services, such as garbage collection, but IoT sensors can improve the services by alerting relevant public works workers when certain garbage cans are full. Smart garbage bins play an important role in environmental growth and preservation. They are equipped with wireless ultrasonic fill-level sensors that detect when the bin is full. The percentage level data is delivered to a cloud-based IoT platform for monitoring and analytics purposes. It can also detect the amount of trash in the garbage bin. Even if people want to dispose of their trash, the lid will not open if the garbage bin is full. The smart bin can also segregate plastic and non-plastic wastes.

\section{LITERATURE REVIEW}

A smart garbage management system was implemented by the "IOT-based Waste Management System for Smart Cities." An Arduino UNO board with GSM modem, ultrasonic sensor and weight sensor is used to create a smart bin. The bin's weight is calculated using the weight sensor. The ultrasonic sensor is used to determine the status of the bin, with a $10 \mathrm{~cm}$ threshold limit. The ultrasonic sensor will trigger the GSM modem, which will alert the appropriate expert when the garbage reaches the limit level. After that, the expert delivers the message to the various administrators. If the trashcan is not cleaned within a certain amount of time, the record is submitted to a higher authority, who can take necessary action against the contractor in question.

An IoT-based smart garbage system (SGS) is proposed in "Smart Garbage Bin for Efficient Food Waste Management" to reduce the quantity of food waste. In an SGS, wireless mesh networks allow battery-powered smart garbage bins (SGBs) to communicate with one another, and a router and server gather and analyze the data for service provisioning. The SGS incorporates a variety of IoT strategies that prioritize user convenience while also extending battery life through two types of energy-efficient SGB operations: standalone and cooperation-based operations. For a year, the suggested SGS was used as a pilot project in the Gangnam district of Seoul, Republic of Korea. According to the findings, the average quantity of food waste decreased by $33 \%$.

A garbage collecting mechanism was proposed in "A Waste Collection Mechanism Based on IoT." To determine the height of the debris, ultrasonic sensors are used. Collection of waste is not required when the garbage container contains only light items such as papers, as these items can be compacted to accept more trash. Weight sensors assist in distinguishing between light and heavy trash, such as paper. Some waste has an intolerable odour, hence priority must be given to the waste bins that emit this type of odour, which can be detected with the MQ Gas sensor. The data from these sensors is sent to a Raspberry $\mathrm{Pi}$ via an Arduino Uno microcontroller. The values of the sensors are continuously monitored. When the value hits the threshold, an alarm is sent to the municipality server, and a garbage clean-up for the related trash can is approved. With the help of this system, the real-time progress of waste collection may be examined.

The "Design and Implementation of a Smart Solid Waste Monitoring and Collection System based on the Internet of Things" offered a solution for municipal authorities' concerns in which a microcontroller is utilized to take action based on the inputs provided by the output/actions of the sensors. Ultrasonic sensing is used to determine the amount of trash in the dustbins. The weight of the dust bin is measured using a force sensor. If the bin is empty or halfway filled, a PIR sensor is used to open the lid automatically. An Android device assists in identifying the location of the trash bin, updating the location, and alerting the appropriate truck to collect the waste. This data, along with the GPS location, is sent immediately to the Amazon cloud web server over GSM.

"IoT-based Smart Garbage Alert System with Arduino UNO" offered a smart alert system for garbage clearance by sending an alert signal to the municipal web server for immediate dustbin cleaning with correct verification based on garbage filling levels. This process is facilitated by an ultrasonic sensor that is connected to an Arduino UNO and checks the level of waste in the trash before sending an alert to the municipal web server. With the use of an RFID Tag, the driver acknowledges the work of discarding the garbage after cleaning the dustbin. An embedded module with RFID and IoT facilitation underpins the entire operation. With the help of this system, the municipality authorities can monitor and follow up on the status of waste collection in real time. An Android application is developed and linked to a web server to send warnings from the microcontroller to the city office and to perform remote monitoring of the cleaning process performed by the workers, eliminating the manual monitoring and verification procedure. The Wi-Fi module is used to send the notifications to the Android application.

\section{METHODOLOGY}

\subsection{Proposed System}

We aim to present a conceptual model of the smart garbage bin. Ultrasonic sensors are used in the proposed system to detect the garbage bin's fill level. In addition, proximity sensors will be used to detect the people coming to dispose of their trash and open the garbage bin automatically. Because the separation of plastic and non-plastic trash is required, inductive sensors and capacitive sensors are used to implement this function. For the segregation purpose, we have separation plates with a partition in the middle. Once the 
garbage falls in the separation plates, the inductive and capacitive sensors will detect the nature of the material. According to that, the particular separator plate will open up downwards, enabling the garbage to easily fall into the trash.
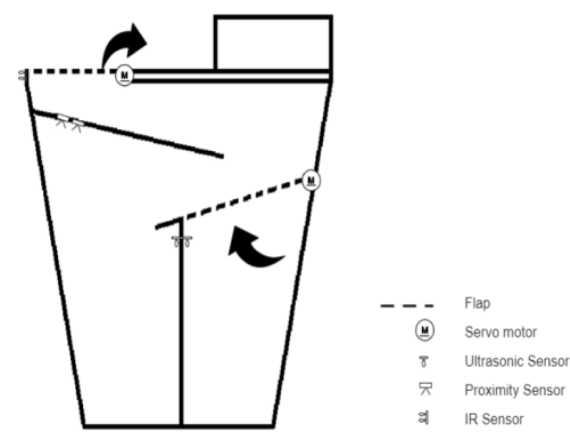

Figure.3.1: Conceptual Smart Bin Prototype

Ultrasonic sensors will be placed between the partitions for getting the fill-level. IR proximity sensors detect persons coming to dump the trash and according to that, the bin lid will open up. The GPS module can be used to show the live location of the garbage bin. The GSM module can be used to send alert messages via SMS to inform the authorities regarding the status of the trash bin. Buzzer alarms can be used as a safety feature. The Arduino board collects data from the sensors, which is subsequently transferred to the ThingSpeak Cloud Platform via the Wi-Fi module. There, we'll be able to see the real-time data of the amount of garbage in the bin graphically, at regular time intervals. The platform will also show the live location of the bin. This project will be developed in such a way that it can be implemented in the campus premises. Further developments can also be made so that it will be made to operate in a much larger area like a city.

\subsection{System Architecture \& Implementation}

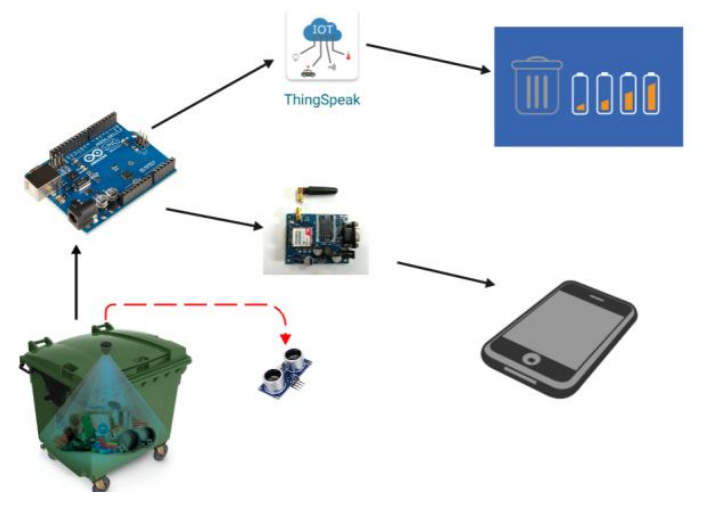

Figure.3.2.1: Architecture of the Proposed System

The figures 3.2.1 \& 3.2.2 gives insights into the working of our conceptual smart electronic bin. It consists of ultrasonic sensors for detecting the percentage fill-level of the garbage bin, IR proximity sensors for the opening and closing of the bin lid, which is done when the sensor detects the people coming to dispose of the garbage, inductive and capacitive sensors for segregation of plastic and non-plastic wastes, the Arduino Uno microcontroller, Wi-Fi module, GPS module, GSM module, servo motors and a battery for power supply. First of all, the segregation of plastic and non-plastic wastes are carried out with the help of the inductive and capacitive sensor which works in accordance with the movement of the servo motors. Inductive sensors work on the principle of inductance, where a fluctuating current causes an electromotive force in the target object. Capacitive sensors measure the changes in capacitance. An electric field is formed when a voltage is supplied, causing positive and negative charges to gather on each item. If the detected waste is a plastic, then the lid of the plastic waste section will open up. Else, the lid of the non-plastic waste section will open. Ultrasonic sensors work by emitting a sound wave that is above the human hearing range. The sensor's transducer functions as a microphone, receiving and transmitting ultrasonic sound. The sensor measures the time between sending and receiving an ultrasonic pulse to determine the distance to a target, and that distance will be converted to the percentage value by making certain changes in the program code. The GPS module can be employed in the system to show the live location of the bin. The GSM module will be used to send alert messages to the monitoring authorities to make them aware of the current status of the bin, so that they can take necessary actions to control excessive waste generation by cleaning them, time to time. The buzzer alarm can be kept as an additional feature in case there are any issues with the bin. The various sensor values are then given to the Arduino Uno for processing and after that, the processed information will be sent to the ThingSpeak cloud platform via the Wi-Fi module. ThingSpeak is a cloud-based IoT analytics software that lets you aggregate, visualize, and analyze live data streams. ThingSpeak delivers real-time visualizations of data sent to it by the devices. With the ability to run MATLAB code in ThingSpeak, we can analyze and handle data as it comes in real time. The ThingSpeak platform will display the real-time data of the garbage inside the bin in graphical form.

The connecting of individual sensors with the microcontroller was the first step in the implementation process. The ultrasonic sensors for distance values, servo motor for exact angular rotation, and Wi-Fi module for wireless communication and range, were all put to the test. The data and information collected and processed by the Arduino are delivered from the microcontroller to the ThingSpeak IoT platform via the ESP8266 NodeMCU bridge component. The master controller will be able to send data across the IoT platform with the help of this Wi-Fi module. The data will be read and written by the IoT platform, and the graph will be plotted. IoT data analytics can be performed with this data, and the nature of trash disposal can be investigated. The status of the smart bin will be displayed in the cloud.

The status of the bin will be updated in real- time because this is a real-time monitoring system. The person-in-charge is notified using a miniature SIM800L cellular module that enables for GPRS transfer, sending and receiving SMS, and 
for making and receiving voice calls. On the back, there is a SIM card slot. Any $2 \mathrm{G}$ micro SIM card that has been activated will function fine. The bin's geographical location is determined using the NEO-6M GPS module with EPROM. GPS modules are made up of tiny computers and antennas that receive data directly from satellites via specific radio frequencies.

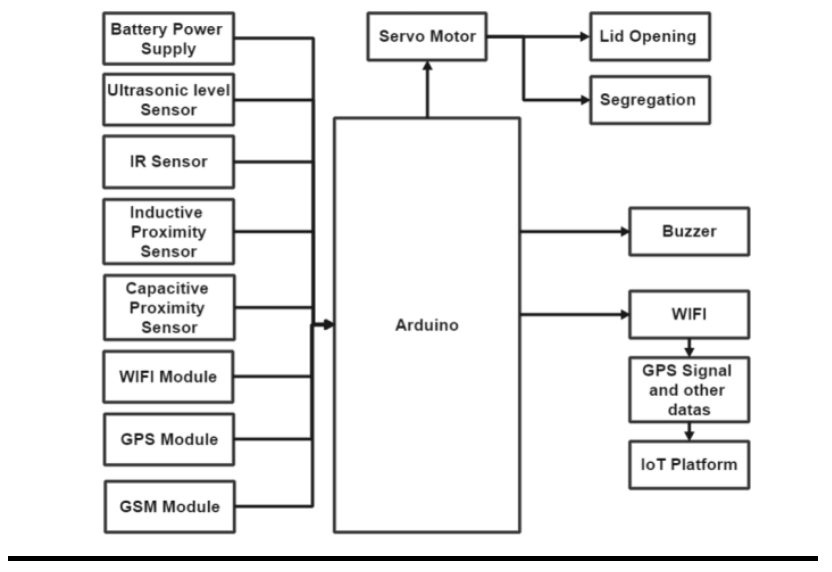

Figure.3.2.2: Architectural Block Diagram

\section{RESULT}

The system is built using a dustbin, ultrasonic sensors, proximity sensors, capacitive sensor, inductive sensor, GPS module, GSM module, servo motor and buzzer alarm. Proximity sensor detects the user and opens the lid.

\section{BIN DATA}

100

Level

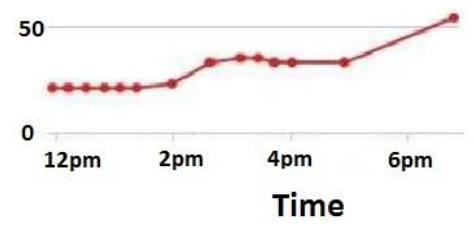

Figure.4: Expected Graph on ThingSpeak Cloud Platform

Opening of the lid is done using a servo motor interfaced with the proximity sensor. Once the lid is opened, the segregation lid will separate plastic and non-plastic waste. This is done with the help of capacitive sensors and inductive sensors. Plastic waste is dumped towards the right side and non-plastic is dumped towards the left side of the bin. Once the waste bin is full, the lid will not open. The GPS module will show the geographical location of the bin. The GSM module will be used for sending and receiving SMS, and making and receiving voice calls to notify the person in charge. The sensor data taken by the ultrasonic sensor will be sent to the Arduino board, from where it goes to the ThingSpeak Cloud Platform. The status of the bin is shown on the ThingSpeak Platform. ThingSpeak was designed to work from iOS and Android smartphones and popular browsers. We will get a graph as shown in the figure.5, which denotes the level of the waste bin at each time interval. The authority (Admin) can see the status of the bin and give instructions to the cleaner. Thus, the authority can take quick actions by cleaning the bin as soon as possible. The table 1 given below gives insights about the results obtained at various test cases.

Table.1: Smart Garbage Bin Status Identification \& Experimental Results

\begin{tabular}{|c|c|c|c|c|c|}
\hline $\begin{array}{c}\text { Test } \\
\text { Cases }\end{array}$ & $\begin{array}{c}\text { Bin } \\
\text { Condition }\end{array}$ & $\begin{array}{c}\text { Cloud } \\
\text { Status }\end{array}$ & Buzzer & LED & $\begin{array}{c}\text { SMS } \\
\text { Sent }\end{array}$ \\
\hline Empty & Filling & $0 \%$ & No & No & No \\
\hline Medium & Filling & $50 \%$ & No & No & No \\
\hline Full & Bin Full & $100 \%$ & Yes & Yes & Yes \\
\hline $\begin{array}{c}\text { Threshold } \\
\text { Crossed }\end{array}$ & $\begin{array}{c}\text { Almost } \\
\text { Full }\end{array}$ & $85 \%$ & Yes & Yes & Yes \\
\hline
\end{tabular}

\section{CONCLUSION}

This project used a smart electronic dustbin to construct a real-time waste management system that senses the dustbin's fill level to determine if it is full or not. This model raises awareness of the importance of keeping our garbage cans clean. Various sensors and an open source platform were used to create the waste bin. ThingSpeak is the IoT platform that has been used. The system will produce accurate reports, enhancing its efficiency. The dustbins will be emptied when they become full, resulting in an improved infrastructure, and improved hygiene. The project's future development will focus on resource allocation optimization and data analysis for decision support system users.

\section{LIMITATION AND FUTURE STUDIES}

An integrated system consisting of the Wi-Fi module, NodeMCU, and an ultrasonic sensor is introduced in this project for efficient and cost-effective garbage collecting. The new system has a better database for garbage pickup times and amount of waste at each location. We will be able to avoid the overflowing of waste from the trash bin by implementing this project. Our work is a small, but an effective step toward cleanliness, and we hope that this report will inspire others to do the same. One of our project's constraints is that the implementation is limited only to a single bin. We will be able to integrate several bins, each with its own unique ID, in the near future, which can be done by implementing the IoT principles. The memory size of the sensor nodes utilized in the dustbins are also restricted. SQL 
technology can be used to create databases for each bin. An automated system that can pick up the garbage in and around the bin, sort it, and place it in the appropriate trash bin can be constructed. A GPS module paired with an ultrasonic sensor for obstacle avoidance can be used to plan a route.

\section{REFERENCES}

1. A Proposed IoT-Enabled Smart Waste Bin Management System and Efficient Route Selection, Journal of Computer Networks and Communications Volume 2019, Article ID7043674.

2. Miss. Megha S. Chaudhari, Mrs. Bharti Patil, Mrs. Vaishali Raut, IOT-based Waste Management System for Smart Cities, Proceedings of the Third International Conference on Computing Methodologies and Communication (ICCMC 2019).

3. I. Hong, S. Park, B. Lee, J. Lee, D. Jeong, and S. Park, IoT-based Smart Garbage System for Efficient Food Waste Management, ScientificWorldJournal., Vol. 2014, p. 646953,Aug. 2014.

4. IntelliBin (The Smart Trash Bin) Hackster.io.[Online].Available:https://www.hackster.io/t eam-rocket/intellibin-the-smarttrash-binb44b5c.[Accessed:22 Oct-2017].

5. A. Mohan, S. Johar, and S. Mini, A Waste Collection Mechanism based on IoT, in 14th IEEE India Council International Conference (INDICON), 2017, pp. 2-6.

6. Aaditya Jain, Ranu Bagherwal, Design and Implementation of a Smart Solid Waste Monitoring and Collection System based on Internet of Things, 8th ICCCNT 2017 July, IIT Delhi.

7. N. S. Kumar, B. Vijayalakshmi, R. J.Prarthana, and A. Shankar, IOT-based Smart Garbage Alert System using Arduino UNO, in 2016 IEEE Region 10 Conference (TENCON),2016, pp. 1028-1034.

8. Murugaanadam, S.Ganapathy and Balaji R, Efficient IOT Based Smart Bin for Clean Environment, International Conference on Communication and Signal Processing in April 2018.

9. Rajkumar Joshil and Sirajuddin Ahmed, Environmental Chemistry, Pollution \&amp; Waste Management, Cogent Environmental Science(2016).

10. S.V. Kumar, T.S.K., A.K.K.a.M.M., Smart Garbage Monitoring and Clearance System using Internet of Things, IEEE International Conference on Smart Technologies and Management for Computing, Communication, Controls, Energy and Materials (ICSTM), 2017.

11. N.M.Yusof, M.F. Zulkifli, M. Yusof, A.Azman, Smart Waste Bin with Real-Time Monitoring System, International Journal of Engineering \& Technology, 7 [2.29] [2018] 725-729.

12. S. Zavare, R. Parashare, S. Patil, Pooja Rathod \& V.Babanne Smart City Waste Management System Using GSM, IJCST - Volume 5 Issue 3, May June2017.

13. Gopal Kirshna Shyam, Sunil kumar S. Manvi, Priyanka Bharti, Smart Waste Management using Internet-of-
Things, 2017 2nd International Conference on Computing \& Communications Technologies (ICCT).

14. G.Prajakta, J. Kalyani, and M. Snehal, Smart Garbage Collection System in Residential Area, IJRET: International Journal of Research in Engineering and Technology, Volume: 04, Issue: 03,pp.122-124, March 2015.

15. P.A.Thole, N.J.Dhage, A.D.Gole, K.D.Kalbhor, IOT Based Smart Waste Management System, IJSRED: International Journal of Scientific Research and Engineering Development- Vol. X Issue X, Year- 2017. 